\title{
A Hidden Group Structure for the Integrals of the Benney System
}

Boris A Kupershmidt

The University of Tennessee Space Institute

Tullahoma, TN 37388, USA

E-mail: bkupersh@utsi.edu

Received October 23, 2008; Accepted November 25, 2008

\begin{abstract}
The integrals of the Benney system are shown to possess a group structure. The KP hierarchy breaks the group law down.
\end{abstract}

\section{Introduction}

The remarkable $2+1$-dimensional integro-differential free-surface hydrodynamical system derived by Benney [1],

$$
\begin{aligned}
& u_{t}=u u_{x}+g h_{x}-u_{y} \int_{0}^{y} u_{x} d y^{\prime} \\
& h_{t}=\left(\int_{0}^{h} u d y\right)_{x},
\end{aligned}
$$

was shown by him to result in a purely differential evolutionary system

$$
A_{n, t}=A_{n+1, x}+g n A_{n-1} A_{0, x}, \quad n \in \mathbf{Z}_{\geq 0},
$$

for the moments

$$
A_{n}=\int_{0}^{h} u^{n} d y, \quad n \in \mathbf{Z}_{\geq 0}
$$

Here $u=u(x, y, t)$ is the horizonal component of velocity at time $t$, at the point, $x, y ; 0 \leq$ $y \leq h=h(x, t), h$ being the elevation of the free surface over the flat bottom $\{y=0\}$; subscripts $x$ and $t$ denote the corresponding partial derivatives.

Benney showed that the system (1.2) is integrable: it has an infinite sequence of polynomial conserved densities $H_{n} \in A_{n}+\mathbf{Z}\left[A_{0}, \ldots, A_{n-2}, g\right]$ :

$$
\begin{aligned}
& H_{0}=A_{0}, \\
& H_{1}=A_{1}, \\
& H_{2}=A_{2}+g A_{0}^{2}, \\
& H_{3}=A_{3}+3 g A_{0} A_{1}, \\
& H_{4}=A_{4}+4 g A_{0} A_{2}+2 g A_{1}^{2}+2 g^{2} A_{0}^{3}, \\
& H_{5}=A_{5}+5 g A_{0} A_{3}+5 g A_{1} A_{2}+10 g^{2} A_{0}^{2} A_{1}, \ldots
\end{aligned}
$$


Subsequently, Manin and myself showed $[5,6]$ that the Benney integrals are in involution with respect to the Hamiltonian structure

$$
B_{n m}=n A_{n+m-1} \partial+\partial m A_{n+m+1}, \quad \partial=\partial / \partial x .
$$

Denote the map sending the $A$ 's to the $H$ 's by $\varphi^{g}$ :

$$
\boldsymbol{H}=\varphi^{g}(\boldsymbol{A})
$$

The purpose of this note is to show that the map $\varphi^{g}$ represents, in fact, a group law:

$$
\varphi^{h} \circ \varphi^{g}=\varphi^{g+h} .
$$

This formula is proven in the next Section. Section 3 discusses a more general perspective, in particular why the law breaks down when one allows the dispersion in, i.e., for the KP flow \#2, whose quasiclassical limit is the Benney system (1.2).

\section{The group law}

Let $u$ be the generator of the ring $K\left(\left(u^{-1}\right)\right)$,

$$
K=\boldsymbol{Q}[g][\boldsymbol{A}],
$$

and consider the equation on $v$ :

$$
g^{-1} u=v+\sum_{i=0}^{\infty}(g v)^{-i-1} A_{i}
$$

Solving this equation, let's write

$$
v=g^{-1} u-\sum_{i=0}^{\infty} H_{i} u^{-i-1}
$$

Thus,

$$
v_{t}=-\sum H_{i, t} u^{-i-1} .
$$

Let us verify that the $H_{n}$ 's are exactly the conserved densities of the Benney system (1.2). Proceeding exactly like in [5], we first verify that

$$
H_{n} \in A_{n}+\boldsymbol{Q}\left[A_{0}, \ldots, A_{n-1}, g\right] .
$$

Next, differentiating $(2.2 a)$ with respect to $u, t$, and $x$, and combining the results, we find that

$$
v_{t}=\left(g \frac{v^{2}}{2}+A_{0}\right)_{x},
$$

as expected. 
We now change perspective and look at the formulae (2.2), as simply a pair of inversion formulae: the $A_{i}$ 's and the $H_{i}$ 's are considered as free variables, without any dependence upon parameters such as $x$ and $t$. Thus, $\boldsymbol{H}=\varphi^{g}(\boldsymbol{A})$.

Set, for the map $\varphi^{h}(\boldsymbol{H})$, the pair of inversion formulae

$$
\begin{aligned}
& h^{-1} x=z+\sum_{i=0}^{\infty}(h z)^{-i-1} H_{i}, \\
& z=h^{-1} x-\sum_{i=0}^{\infty} x^{-i-1} G_{i},
\end{aligned}
$$

and for the map $\varphi^{h+g}(\boldsymbol{A})$, the pair

$$
\begin{aligned}
& (h+g)^{-1} x=y+\sum_{i=0}^{\infty}[(h+g) y]^{-i-1} A_{i}, \\
& y=(h+g)^{-1} x-\sum_{i=0}^{\infty} x^{-i-1} \tilde{G}_{i} .
\end{aligned}
$$

We want to show that

$$
G_{i}=\tilde{G}_{i}, \quad i \in \mathbf{Z}_{\geq 0} .
$$

Comparing formula (2.6b) and (2.7b), (2.8) becomes:

$$
z-h^{-1} x=y-(h+g)^{-1} x,
$$

or

$$
z=y+\frac{g}{h(h+g)} x .
$$

Thus, our original system $\{(2.2,6,7)\}$ is reduced to $\{2.2,6 a, 7 a, 10)\}$.

Next, set

$$
u=h z[\text { by }(2.10)]=h y+\frac{g}{h+g} x .
$$

The equations $(2.2 b, 6 a)$ are then replaced by

$$
g^{-1} u-v=h^{-1} x-z,
$$

or, with the help of formula (2.10), by

$$
v=\frac{h+g}{g} y .
$$

Our new system is now $\{(2.2 a, 7 a, 11,13)\}$.

Since, by formula (2.13),

$$
g v=(h+g) y,
$$


the equations $(2.2 a)$ and $(2.7 a)$ are compatible iff

$$
g^{-1} u-v=(h+g)^{-1} x-y,
$$

which formulae $(2.11,13)$ imply at once. We are done.

Remark 2.16. With hindsight available, the symmetry $\boldsymbol{A} \rightarrow-\boldsymbol{H}$ described in $\S 1.4$ in [5] is a particular case

$$
g=-h=1
$$

of our general group law (1.6).

\section{Generalizations}

As shown by Manin and Lebedev in [7], the Benney hierarchy constructed in [5,6], is the zero-dispersion limit of the KP hierarchy (see, e.g., [2]). The Benney flow (1.2) is the quasiclassical limit of the second KP flow ( $g$-scaled)

$$
\begin{aligned}
& A_{n, t}=\frac{1}{2} g A_{n}^{(2)}+A_{n+1}^{(1)}+\sum_{r \geq 0}\left(\begin{array}{c}
n \\
r+1
\end{array}\right)(-1)^{r} A_{n-1-r} A_{0}^{(r+1)} g^{r+1}, \\
& (\cdot)^{m}=\partial^{m}(\cdot) .
\end{aligned}
$$

Surprisingly enough, the group law (1.6) breaks down under the influence of dispersion. (This can be seen already for the integral $H_{4}$ ). I conjecture that the underlying reason is that the KP hierarchy is not the right hierarchy whose quasiclassical limit is the Benney hierarchy: the KP ansatz is stripped so much off its natural gauge degrees of freedoms as to become too rigid. This conclusion can be arrived at by analyzing Wilson's second construction [8].

On the other hand, in purely hydrodynamic (zero-dispersion) case, the group law appears to hold (sometimes in modified form) for many hydrodynamical chains described in $[3,4]$. This subject is now under development.

\section{References}

[1] Benney, D. J., Some Properties Of Long Nonlinear Waves, Stud. Appl. Math. L11 (1973) 45-50.

[2] Dickey, L. A., Soliton Equations and Hamiltonian Systems. Second edition. World Scientific Publishing Co., Inc., River Edge, NJ (2003).

[3] Kupershmidt, B. A., Deformations of Integrable Systems, Proc. Roy. Irish Acad. 83 A No. 1 (1983) 45-74.

[4] Kupershmidt, B. A., Normal and Universal Forms in Integrable Hydrodynamical Systems, in Proc. of NASA Ames-Berkeley Conf. on Nonlinear Problems in Optimal Control and Hydrodynamics, L. R. Hunt and C. F. Martin Ed-s, Math. Sci. Press (1984) 357-378. 
[5] Kupershmidt, B. A. and Manin, Yu I., Long-Wave Equation with Free Boundaries. I. Conservation Laws, Funct. Anal. App. 11:3 (1977) 31 - 42 (Russian); 188-197 (English).

[6] Kupershmidt, B. A. and Manin, Yu I., Equations of Long Waves with a Free Surface. II Hamiltonian Structure and Higher Equations, Funct. Anal. Appl. 12:1 (1978) 25-37 (Russian); 20-29 (English).

[7] Lebedev, D. R., and Manin, Yu. I., Conservation Laws and Lax Representation of Benney's Long Wave Equations, Phys. Lett. A 74 (1979 154-156.

[8] Wilson, G., On two Constructions of Conservation Laws for Lax Equations, Quart. J. Math. Oxford Ser. (2) 32 (1981) 491-512. 